\title{
Educación en tiempos de COVID-19: Aprender desde la diferencia
}

\author{
Erica Tejedor Fuentes ${ }^{1}$
}

\section{Resumen}

La educación es vista como el vehiculo idóneo para cambiar las realidades sociales, pero, es uno de los sistemas con mayores brechas por cerrar para garantizar la calidad de los procesos que la intervienen. Por ello, este trabajo tiene como propósito mostrar cómo a causa del COVID-19 o Coronavirus, se han aflorado algunas de las desigualdades que subsisten en el interior de la educación. Esta reflexión se enmarca en el contexto colombiano, para lo que se bizo necesaria una revisión literaria exhaustiva mientras que se aplicó como método aunador la observación y el análisis. Llegando al consenso de que el sistema educativo colombiano necesita de estrategias que contribuyan a mitigar las problemáticas presentes y futuras provocadas por el COVID-19 las cuales se centran en la eminente desigualdad social.

Palabras clave: Covid-19; desigualdad social; educación; inclusión educativa; inclusión social

\section{Education in times of COVID-19: Learning from difference}

\begin{abstract}
Education is seen as the ideal vehicle to change social realities, but it is one of the systems with the largest gaps to close to guarantee the quality of the processes involved. Therefore, this work aims to show how due to COVID-19 or Coronavirus, some of the inequalities that subsist within education have emerged. This reflection is framed in the Colombian context, for which an exhaustive literary review was necessary while observation and analysis were applied as a unifying method. Reaching the consensus that the Colombian educational system needs strategies that contribute to mitigating the present and future problems caused by COVID-19, which focus on the eminent social inequality.
\end{abstract}

Keywords: Covid-19; Social inequality; Education; Educational inclusion; Social inclusion

\section{Introducción}

La educación ha sido sometida a diversos cambios a raíz de múltiples sucesos, entre ellos la globalización y la sociedad del conocimiento. Sin embargo, en la actualidad, ha de atravesar por la situación más compleja de los últimos decenios, la pandemia a nivel mundial originada por el COVID-19. Esta obligó a profesores y a estudiantes a convertir sus aulas físicas en aulas virtuales. Pero, cha de ser inclusivo el sistema educativo en el amplio sentido de la palabra? ¿cómo han sido abordadas las diferencias para que este sea equitativo?

Por consiguiente, Mokate (2002) asegura que la Comisión Económica para América Latina y el Caribe (CEPAL) ha de aseverar que la equidad "define las mismas condiciones y oportunidades para todas las personas sin distinción, solo adaptándose en casos particulares, a los que plantean objetivos para avanzar hacia una sociedad más justa”. Así, el Banco Mundial

\footnotetext{
${ }^{1}$ Erica Tejedor Fuentes, Docente investigadora Universidad del Atlántico en Colombia.

Correo electrónico: eptejedor5@misena.edu.co.
} 
(2011) citado por (Muñoz-Pogossian y Barrantes, s.f.) hace alusión a que la desigualdad debe ser vista como aquella "dispersión de una distribución en el ingreso, consumo, o en algún otro indicador relacionado con el bienestar". De este modo es posible entender que la sociedad debe asumir que está en el derecho de acceder a todo cuanto ofrece el sistema nacional en materia de equidad. No obstante, la sociedad ha sido expuesta a diversas marginaciones en las que se asegura el cumplimiento de las políticas que buscan sanar las diferencias existentes. Puesto que más allá de vivir en un Estado social y económico equitativo, se promulga a gran escala la desigualdad; quien conlleva a su vez, no solo a la exclusión social sino también, a la exclusión educativa.

Más adelante, el Banco Mundial (2014) citado por (Muñoz-Pogossian y Barrantes, s.f.) expone que la inclusión social es un "proceso de empoderamiento de personas y grupos para que participen en la sociedad y aprovechen sus oportunidades. Da voz a las personas en las decisiones que influyen en su vida a fin de que puedan gozar de igual acceso a los mercados, los servicios y los espacios políticos, sociales y físicos". En contraste, la CEPAL (2014) citado por (Muñoz-Pogossian y Barrantes, s.f.) advierte que la inclusión social debe ser entendida como un "proceso por el cual se alcanza la igualdad, y como un proceso para cerrar las brechas en cuanto a la productividad, a la educación y el empleo, la segmentación laboral, y la informalidad, que resultan ser las principales causas de la inequidad". Dicho proceso, a razón del persistente COVID-19, dejó ver que todavía existen barreras litigantes no solo a nivel socioeconómico sino además, a nivel de educación. Lo cual demuestra que las exclusiones sociales permean en estos sistemas provocando afectaciones que no dan espera para ser sanadas. Desde esta perspectiva, este ensayo tiene como objetivo mostrar cómo el COVID19 ha aflorado y profundizado en algunas de las desigualdades ya existentes en el sistema educativo colombiano. Consecuentemente, este esbozo quiere servir como reflexión en el campo de la investigación educativa.

\section{Contexto educativo colombiano}

El Ministerio de Educación Nacional (MEN) postula que la educación en Colombia es un derecho y una prioridad por la cual el gobierno de velar a fin de que los ciudadanos gocen plenamente de él. Esto de conformidad con lo proclamado por la Constitución de 1991 y la Ley General de Educación de 1994; pues, es necesaria para el desarrollo personal y para el beneficio de la sociedad. A su vez, en consonancia con otros países como México, con base en el PND, Colombia, pretende que para el año 2030 la educación obligatoria circunscriba la educación media (MEN, 2015).

Las políticas públicas generadas alrededor de 2016, han dado su cuota a que la educación colombiana haya ampliado su participación significativamente, en especial en los niveles que dieron muestras de la disminución del número de matriculados. Ya que a diferencia del 2000 y 2002, entre 2012 y 2013, hubo un aumento sobre el ingreso de estudiantes a la primaria del $36 \%$ al 45\%; por su parte, en básica secundaria pasó del 59\% al 70\% y, en la educación media se evidenció que incrementó del 30\% al 41\%. Por otro lado, es preciso mencionar que se han efectuado una serie de medidas en las que se ha invertido no solo en infraestructura sino también, en la prestación del servicio de educación pública gratuita, conllevando a que estos indicadores logren mantenerse (UNESCO-UIS, 2015).

Seguidamente, el MEN, apunta que una serie de factores tienen gran incidencia en el aumento de las tasas de deserción escolar en Colombia, entre los que se inscriben la deficiente transición 
entre niveles y el significativo número de niños y jóvenes que no ingresan al sistema educativo. A la vez, hace hincapié en que a pesar de que la tasa de deserción desde el preescolar hasta la básica secundaria ha disminuido entre 2002 y 2013, la proporción de niños que no ingresaron a cursar la primaria aumentó un 4\% en 2010 (OCDE/CAF/CEPAL, 2014). Situación que sin duda, gracias a la pandemia por COVID-19 ha de reflejarse drásticamente en la tasa actual. Pues "la educación básica secundaria sigue siendo el eslabón más débil del sistema educativo colombiano" debido a que la tasa anual continúa superando a la de educación primaria (MEN, 2015; UNICEF, 2012). Lo que atribuye a que Colombia con un 36\%, supere el doble de lo promediado por la OCDE (13\%); situación semejante es la de México quien proyecta un 35\% (OCDE, 2015).

Según el MEN (2016, p. 32) "la evidencia sugiere que una infraestructura y unos materiales educativos inadecuados afectan el acceso y cumplimiento de logros. Este es un problema en todos los niveles del sistema". A razón de esto, se puede apreciar el hecho de que si antes de que se proclamara pandemia por COVID-19, no había sido posible enfrentar esta problemática, el telar, una vez iniciada la etapa educativa durante la emergencia sanitaria, se extiende aún más acarreando una serie de interrogantes que reclaman prontas respuestas.

Partiendo de lo analizado por el Departamento Nacional de Planeación (DNP), quien asegura que una cantidad significativa de escolares colombianos no están sumamente preparados para incursionar en la educación superior o al mercado laboral. Esto con base a que el $27 \%$ de las personas que presentaron el examen nacional SABER 11 en 2013 situaron sus resultados en el nivel bajo o inferior, demostrando que poseen falencias de alto grado para la retención y el éxito en la educación superior (DNP, 2015). Es aquí en donde cabe detenerse a pensar en que el sistema educativo de Colombia no está lo suficientemente ajustado a las necesidades que coexisten. Lo que advierte una imperante necesidad de reestructuración en el interior del mismo, ya que si el panorama presentado antes de la pandemia es desalentador, el actual se reviste de mayores retos que no dan espera para ser cumplidos. De no encontrarse una solución que coadyuve a que los niños, niñas y jóvenes lleguen a presentar estos exámenes con la preparación suficiente durante y después del COVID-19, estos tendrán menos oportunidades de ingresar a la educación superior y al ámbito laboral no precario gracias a que no han adquirido las competencias que cada vez son más demandantes. Esta situación solo ha de demostrar que la exclusión social y educativa ha de incrementarse para aquellos que poseen menos recursos económicos.

Por su parte, el gobierno nacional ha puesto su mirada no solo en los niños que provienen de comunidades en condición de pobreza, asimismo, introdujo medidas que se adaptan a satisfacer las necesidades de grupos vulnerables como las comunidades étnicas, los niños con Necesidades Educativas Especiales (NEE), las personas que han sufrido afectaciones por la violencia y las poblaciones rurales. Aunque, componentes dados al origen socioeconómico, la ubicación geográfica, la etnia y el género, siguen siendo condicionantes a grandes rasgos de las oportunidades educativas de los colombianos (MEN, 2016, p. 34). Con esto se insiste en que las políticas públicas continúan siendo insuficientes, y que los componentes mencionados más allá de verse como un obstáculo deberían ser el eje que permita focalizar en suma medida que las desigualdades se acrecientan en pos de evitar que estas poblaciones reciban una educación de calidad, lo cual se muestra como una urgencia y no como un simple deseo.

Colombia busca transformar la educación a través de una descentralización efectiva, pero, aun cuando "fue uno de los primeros países en América Latina en empezar a descentralizar su 
sistema educativo y es el Estado unitario más descentralizado de la región. El proceso fue rápido y de amplio alcance, pero aún está incompleto” (p. 42). De manera que no está de más hacer imbricaciones acerca de que pese a que los objetivos propuestos pretenden una proyección amplia en cuanto a la transformación educativa del país, la meta no ha sido alcanzada gracias a que los esfuerzos hechos son insuficientes o poco asertivos.

\section{La inclusión, exclusión e integración en el contexto educativo colombiano}

Silver (1995) refrenda que la inclusión social se define desde diversos ángulos que dan trascendencia a una efectiva reducción de la pobreza que al mismo tiempo abarcan factores de exclusión más sutiles derivados de carencias económicas y sociales. Entre tanto, Behrman, Gaviria y Szekely (2003) entienden como exclusión social "la negación del acceso igualitario a las oportunidades que determinados grupos de la sociedad imponen a otros". Desde este punto, se concibe que la educación es uno de esos factores que han sido conducidos a la exclusión social. Debido a que desde antes y durante la pandemia por COVID-19, los estudiantes de los estratos más bajos no tienen acceso a educación de calidad; más aún, luego de que se diera como metodología emergente el crear aulas virtuales, ya que estos carecen de recursos económicos para costear un plan de servicio internet y equipos tecnológicos como tabletas, celulares de alta gama o un computador. En este sentido, Tylly (1999) afirma que la inclusión social logra distinguirse y completar el concepto de igualdad, pues, esta revela la existencia y durabilidad de ciertas desigualdades.

Hablar de igualdad e inclusión al interior de la educación en Colombia es un tanto complejo, por lo que las realidades basadas en una marcada desigualdad permanecen en el tiempo haciéndose cada vez más vastas. Mientras que los entes encargados de brindar soluciones se hacen los de la vista gorda intentado detener con unos cuantos sacos de arena las incontenibles aguas de una presa que amenaza con desbordarse.

Según la OACDH (2012) "la dignidad humana es el fundamento mismo de los derechos humanos, y está inextricablemente vinculada con los principios de igualdad y la nodiscriminación". Viéndolo de esta forma, la igualdad y la no-discriminación "exigen que los Estados identifiquen a los grupos vulnerables y desfavorecidos de la sociedad y concedan prioridad a garantizar que esos grupos disfruten de los derechos humanos en pie de igualdad". En Colombia de algún modo se lleva a cabo esta tarea, sin embargo, de nada sirve identificar a estas poblaciones si no se han de solventar de manera significativa las necesidades de educación de calidad, vivienda digna, salud y empleo no precario. Simplemente sería como resaltar a groso modo que las desigualdades van en crecimiento y que los pocos esfuerzos adoptados integran a unos cuantos dejando a los demás a la espera.

De este modo, a la luz del análisis que se realizó sobre Desarrollo Social Inclusivo, la CEPAL (2015) atribuye que para direccionar un avance hacia la igualdad es propicio empezar por reconocer y caracterizar la matriz de la desigualdad social de América Latina y el Caribe, incorporando esas dimensiones, de manera que se llegue a la formulación y estructuración de los mecanismos de implementación, monitoreo y evaluación de las políticas públicas. Ya que si no se logran resultados, la desigualdad y la exclusión social seguirán percibiéndose por los ciudadanos como el fomento de pésimas acciones que garantizan una sociedad en la que la democracia no concibe a todos por igual. Mientras tanto, "la pobreza y la desigualdad siguen siendo retos considerables para Colombia y son aún más pronunciados entre una región y otra" (MEN, 2016, p. 23). Tal es el caso de aquellos que no cuentan con un sistema de

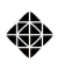

Yeiyá 
transporte y/o carreteras que les permitan movilizarse hasta los lugares que les han de prestar los servicios de salud, educación e incluso hasta sus empleos. Muchas de estas regiones olvidadas han optado por crear modalidades de transporte altamente peligrosas (como tener que atarse a una cuerda que se sostiene de un árbol para luego ser lanzados hasta el otro extremo) las cuales deben utilizar sí o sí. Es ahí en donde surgen preguntas sobre el manejo que se le da a los dineros destinados para el crecimiento del país y de la sociedad misma. Esta eventualidad se puede apreciar como punto de partida para provocar de alguna manera que la deserción escolar y que el analfabetismo sea una constante en estas comunidades, ya que para ellos llegar diariamente a las aulas de clases se ha convertido en un reto suicida.

Almenara y Córdoba (2009) hacen hincapié en que se han tergiversado los conceptos de inclusión e integración educativa, puesto que, según ellos, se trata de dos enfoques totalmente diferentes en los que existen planteamientos y centros de interés distintos. Gracias a que esto implica una reestructuración en la que se da un proceso de cambio interno, curricularmente hablando, en los sistemas educativos. Por tanto, citan a la UNESCO (2005), quien alude que la inclusión se relaciona con acceso pertinente, la participación activa y los logros de cada uno de los estudiantes; especialmente, de aquellos que se ven expuestos mayormente a la exclusión y a la marginación.

Posteriormente, la misma fuente, propone lo dicho por López (1997, p. 182) en cuanto a que la integración es una acepción afiliada al reduccionismo que tiende a promover el mejoramiento de la educación especial, pero, olvidándose de la educación general. Atendiendo esto, los estudiantes que presentan NEE, como se ha manifestado, exigen una adecuación curricular que les permita adquirir y desarrollar sus habilidades motoras y cognitivas. No obstante, el sistema educativo, al diseñar una aula virtual de modo que se cumpliera con lo dictado por el Gobierno Nacional, para así garantizar la seguridad de cada uno de sus actores en temas de salud, no tuvo en cuenta características como: el que esos educandos contaran con acceso al servicio de internet, que pudieran sostenerlo económicamente; que tuvieran en sus casas algún dispositivo tecnológico para participar de cada una de las clases; que las actividades presentadas brindaran garantías de calidad educativa y; que contaran con el acompañamiento idóneo para que tales actividades cumplieran y satisficieran las necesidades de todos y cada uno de los escolares con NEE.

Es importante entender y promulgar que cada estudiante es diferente, lo cual conlleva a que los procesos de enseñanza aprendizaje se ajusten a lo que realmente necesitan para dar cuenta de la calidad de la educación que se les está entregando; esto por un lado. Además, se ha de decir que aun cuando la población con NEE sea vista como "residentes digitales" (Withe, 2011), su proceso de enseñanza aprendizaje debe girar en función del alcance de metas y objetivos claros para que este sea significativo. Hecho que bien podría relacionarse con el postulado citado por Almenara y Córdoba (2009), en donde se manifiesta que no se puede hablar de una única brecha digital, sino que sería más concreto referirse a brechas digitales, ya que deben ser percibidas como ese elemento multidimensional que incluye barreras de disímiles categorías: económicas, de localización geográfica, de edad, de sexo, de idioma, de educación y procedencia sociológica o cultural, o aquella que se deriva de la heterogeneidad de las personas con diversidad funcional (Cabero, 2004, Bindé, 2005). Dicho de otra manera, es importante tener presente que para garantizar que exista inclusión en su totalidad, es necesario que se sienten los mecanismos a efectuar desde una base sólida que contenga el verdadero palimpsesto de las distintas realidades que se busca saciar. 
En contraposición, Hurtado y Agudelo (2014) aceptan el término de integración a partir de lo propuesto por Fernández (2003) al decir que la integración de niños con NEE, resulta beneficioso no solo para ellos, sino también que promueve el mejoramiento de la calidad educativa, gracias a que aquellos que son integrados en el ámbito de la escuela común han de ocupar un espacio social en el que se establecerán vínculos sostenidos en la satisfacción de las necesidades que cada uno tenga, mientras que se crean espacios de aprendizaje copartícipes.

Sin embargo, más adelante, aclaran que para llegar al término de inclusión escolar, este, tuvo que madurar ya que a pesar de que han pasado 30 años desde su aparición, en Colombia, continúa siendo una disciplina joven que solicita de una mayor evolución. Así, sientan que "las prestaciones educativas especiales son un problema que afecta por igual a los países del norte y a los del sur, que no pueden progresar aisladamente, sino que deben formar parte de una estrategia global de la educación y, desde luego, de nuevas políticas sociales y económicas. Esto requiere una reforma considerable de la escuela ordinaria" (Declaración de Salamanca, 1994). En Colombia, esta población, cada vez va en ascenso, pero, desde el sistema educativo, no se han orientado las estrategias suficientes e idóneas que garanticen el verdadero respaldo que esta necesite. Puesto que la educación inclusiva no ha sido concebida de un todo como "una búsqueda incesante de mejores formas de responder a la diversidad. Se trata de aprender a vivir con la diferencia y de aprender a capitalizar las experiencias derivadas de las diferencias. De tal forma que estas últimas lleguen a considerarse más positivamente como incentivo para fomentar el aprendizaje, tanto de niños como de adultos". Con base en este acepto, se cree propio abordar la educación para la paz, ya que es de carácter imperioso que cada ser humano entienda el significado de aprender a convivir juntos, gracias a que es de gran vitalidad a la hora de construir sociedad. El hecho de determinar aulas en las que se promulgue la diversidad estaría permitiendo que los valores y saberes enseñados a los estudiantes sean puestos en práctica en contextos reales. Pero, hay que mencionar que este debe ser un trabajo en el que se refleje la constancia y la participación de todos los involucrados para que se dé un verdadero aprendizaje. Porque de nada servirá generar inclusión sin una correcta preparación y adecuación de los espacios que se quiere propiciar. De otro lado, tales vínculos solo podrán satisfacer las necesidades de cada escolar cuando se comprenda que el docente es pieza clave en el proceso. Por ello, debe contar con las herramientas y competencias que le den acceso a la creación de estrategias que aporten en un sentido positivo a la significación de una educación de calidad.

Analizando lo anterior, es preciso hacer un llamado a la reflexión debido a que, incluso los docentes, no son capacitados para desarrollar y llevar a cabalidad la tarea de enseñar a los escolares con alguna discapacidad y/o necesidad educativa especial; puesto que desde el currículo universitario, esta situación no es atendida. Es entonces, por lo que los educadores llegan a los salones de clase carentes de recursos para abordar esos contextos desconocidos. Hurtado y Agudelo (2014) presentan el trabajo realizado por Padilla (2011) en Bogotá D. C. El mismo, demuestra que "solo de un $20 \%$ a un $30 \%$ de los docentes dicen hallarse en capacidad para educar a estudiantes con discapacidad. Por tanto surge la necesidad de intervenir sobre los docentes, con el fin de evitar que se incrementen las barreras de la inclusión por parte de ellos". Esto esclarece que desde el currículo universitario no se ha ideado el hecho de proporcionar a los educadores herramientas que les ayuden a desempeñarse en ese contexto desconocido; pues, solo los que escogen la carrera de educación especial y a fines, son quienes tienen derecho a conocer parte de la realidad de trabajar con esta población. Cabe decir que si no se toman medidas que promuevan soluciones eficaces 
con respecto a las falencias halladas, más aun en la actualidad, se ha de vislumbrar que la deserción y el fracaso escolar han de significar el índice más elevado de la tasa anual que se haya visto en los últimos tiempos

\section{Impacto del COVID-19 y contextualización de las medidas gubernamentales en materia de educación}

La llegada del COVID-19 a Colombia impactó en profundidad al sector educativo de forma negativa debido a que obligó a profesores, estudiantes y padres de familia a ajustarse a una realidad llena de incertidumbres, en la que las afectaciones socioemocionales, especialmente en los niños, niñas y adolescentes no se hicieron esperar. El encierro por la cuarentena obligatoria y el tener que recibir clases de una manera diferente a la acostumbrada muy seguramente se constituyen como los detonantes más característicos de la desmotivación que se produjo en ellos. Para los educadores y padres de familia tampoco se concibe una situación fácil de asimilar, puesto que para los primeros el trabajo se triplicó en comparación a lo usual; mientras que los últimos han tenido que hacer las veces de docente sin conocer si quiera cómo funciona la pedagogía. Esto sin contar que muchos de ellos tienen uno o dos hijos con NEE y que carecen de herramientas y de estrategias que les ayuden a sobrellevar las exigencias que se den a causa de las necesidades de sus hijos en términos conductuales, afectivos y de enseñanza aprendizaje. Según la UNESCO (2020) citada por (Bosada, 2020) Estrés, ansiedad y presión son los principales efectos que se produjeron a raíz de lo anterior.

A lo largo de los años, el MEN en conjunto con las entidades gubernamentales y la sociedad civil ha llevado a cabo diferentes políticas públicas con relación a la educación en Colombia. Entre ellas están la concernida para la educación inicial la cual nace como resultado obtenido de un proceso de movilización social, en el que se dieron a conocer las necesidades de hacer un ajuste con el fin de dar un nuevo significado a las oportunidades efectivas que promuevan el desarrollo de la primera infancia colombiana. Dentro de ella se encuentran proyectos como "de cero a siempre", el más reciente, cuyo objetivo es garantizar la atención integral de las mujeres en periodo de gestación, niños y niñas de cero a seis años y la educación preescolar de estos. También se incluye el cuidado de los niños y niñas, y la oportunidad de obtener una alimentación sana que contribuya a su desarrollo y crecimiento (MEN, 2007).

Esta política se logra entender y asumir como una iniciativa que procura mitigar las carencias socioeconómicas y de educación presentadas en los ciudadanos situados en estado de vulnerabilidad y escasos recursos económicos. La misma ha contribuido a que muchos niños y niñas sean tenidos en cuenta al tratar de brindar soluciones ante la exclusión social. No obstante, es preciso decir que los cupos establecidos podrían no alcanzar a cobijar a toda la población infantil. Por lo que se cree que es muy importante revisar aspectos en materia de cobertura para que esta realidad disminuya porcentualmente.

De otro lado, la política se justifica en argumentos relacionados con el desarrollo humano, argumentos científicos, argumentos sociales y culturales, argumentos legales y políticos, argumentos ligados al contexto institucional y programático y en argumentos éticos (MEN, 2007). Porque aún a la fecha no se ha comprendido que brindar una atención integral a la primera infancia debe ser tenido como una prioridad que no da lugar a postergaciones. El desarrollo inicial del ser humano es concebido como la base fundamental para garantizar que este tenga pleno desenvolvimiento en las siguientes etapas de su vida. En los primeros años de vida es en donde las habilidades cognoscitivas, motoras, sociales y afectivas se han de 
construir y de no hacerse con idoneidad se podría incurrir en el aparcamiento de las mismas conllevando al fracaso no solo escolar sino social. Aun cuando Colombia se ha comprometido a cumplir con esta política, consumar este propósito se reconoce como un vasto desafío que requiere de revisiones periódicas que permitan establecer acciones que permeen positivamente en los resultados que se esperan.

Para la educación básica y media también se han estipulado políticas públicas, entre las que se efectúan proyectos como los de etnoeducación. Con estos se quiere dar acompañamiento y fomentar la consecución de los procesos de consulta, participación y concertación activa desde el corazón de cada grupo étnico para que sus necesidades particularidades sociales, culturales, lingüísticas y territoriales tengan respuestas que reduzcan el déficit de no escolarización (MEN, 2019). Crear espacios en los que la diversidad étnica tenga acceso a la educación se concibe como una ardua tarea a la cual se le debe prestar la atención requerida. Ya que si se hace del modo incorrecto, en vez de promover la inclusión de estos grupos en las distintas aulas de clase simplemente se estaría provocando una integración pasiva.

Dentro del proyecto de cobertura se presenta el de atención a la población vulnerable quien integra las poblaciones afectadas por la violencia, niños, niñas y jóvenes trabajadores, la articulación de la oferta educativa con las cajas de compensación, la población iletrada con alto grado de vulnerabilidad y la población con Necesidades Educativas Especiales. Las políticas generadas para estos tipos de población pretenden ofrecer igualdad de oportunidades en la educación de cada uno de sus miembros (MEN, 2020c). Partiendo de la revisión hecha a la descripción sentada por el Ministerio de Educación Nacional, estos proyectos continúan sin respuestas asertivas, debido a que bien podrían considerarse el grueso de las desigualdades marcadas en materia de educación nacional. Intentar llegar a las zonas de difícil acceso para gestionar cambios significativos al interior de sus comunidades reclama revisiones y reestructuraciones que convaliden una inmersión total en aras del cumplimiento de las políticas públicas dispuestas para combatir la exclusión social.

Por otra parte los modelos educativos flexibles, el Proyecto de Educación Rural (PER), transporte escolar, infraestructura y dotación, Familias en acción, el programa de alimentación escolar, el programa de gratuidad educativa y las jornadas educativas complementarias también prometen su cuota en cuanto a fomentar cambios drásticos que contribuyan al reduccionismo de la falta de oportunidades educativas en Colombia. Sin embargo, pese a estos esfuerzos todavía se evidencian inconsistencias intrínsecas y extrínsecas en el sistema educativo nacional que exigen de una mayor rigurosidad.

Sin duda, todas las políticas antes mencionadas buscan un mismo fin, reducir a gran escala la falta de oportunidades para los niños, niñas y jóvenes menos favorecidos con el objetivo de brindarles igualdad, equidad y paridad por medio de la inclusión social y educativa. Pero, la realidad que subyace sigue siendo inminente ante cualquier impulso carente de la fuerza que verdaderamente se requiere. En este punto cabe precisar en que la economía del país da muestras de que no ha sido manejada de la forma más apropiada, lo que ha causado panoramas desalentadores para aquellos que cuyo nivel socioeconómico se sitúa en la pobreza y por debajo de ella. Lo que significa que aunque el Gobierno Nacional ofrezca comedores al interior de las escuelas y transporte escolar, no todos los estudiantes resultan ser beneficiaros. Por lo que por muy mínimo que parezca, las desigualdades y la exclusión social siguen estando a la orden del día. De manera que con la llegada del COVID-19 es posible pensar en que el 
lado más inclinado de la balanza ha logrado acercarse a su base como consecuencia del peso añadido.

\section{Contextualización de las medidas gubernamentales en materia de educación tras la COVID-19.}

El pasado 14 de marzo de 2020 la Ministra de Educación Nacional María Victoria Angulo González emitió la circular No. 19. En la que dio a conocer que la Organización Mundial de la Salud (OMS) declaró como pandemia al COVID-19, por lo que invitó a todos los países a que emprendieran acciones para evitar la propagación y transmisión del virus. Así, la Ministra, aseveró que por medio de la Circular Conjunta No. 11 del 9 de marzo de 2020, los Ministros de Educación Nacional y de Salud y Protección Social determinaron recomendaciones para la prevención, manejo y control en el sector educativo para evitar que el Coronavirus afecte colateralmente al mismo. Esto en consonancia con las directrices de la OMS, la UNICEF y la Cruz Roja Internacional.

Con base en lo prescrito por la OMS el Ministerio de Salud y Protección Social emite la Resolución 385 del 12 de marzo de 2020, a través de la cual consideró prudente declarar la emergencia sanitaria hasta el 30 de mayo de ese mismo año en todo el territorio nacional con el objetivo de implementar medidas que procuraran la contención, el control y la propagación del Coronavirus. La Circular además, cuenta con medidas optadas por el Gobierno Nacional para que las fronteras con la República de Venezuela fueran cerradas ya que estas son situadas como corredores humanitarios que consienten el tránsito de estudiantes residentes de ese país matriculados en todos los niveles de educación colombiana. A su vez, atendiendo a lo sentado en los numerales 5.1 y 5.2 del artículo 5 de la Ley 715 de 2001 en concordancia con el literal d) y el numeral 3 del artículo 148 de la Ley 115 de 1994, se insta al MEN la facultad para coordinar todas las acciones educativas del Estado y de quienes presten los servicios educativos públicos en la Nación.

Lo anterior con el fin de que se adopten medidas preventivas en los establecimientos educativos para garantizar la protección y los derechos fundamentales de la salud en los niños, niñas y jóvenes del país. Las recomendaciones para el manejo y tratamiento del autocuidado también tienen lugar en la circular No. 19. Y se esclarece que si los establecimientos educativos logran cumplir con todos los mecanismos de protección deberán tomar los Fondos de Servicios Educativos para ejecutar estas acciones. Hecho que llama mucho la atención debido a que antes de concebir que se tomen estos fondos debería hacerse un estudio o una auditoria profunda para determinar si realmente estos cuentan con la cantidad suficiente para por lo menos pensar en adquirir lo básico.

Luego de que se buscaran prontas soluciones que permitieran la continuidad de las clases, el pasado 15 de marzo de 2020 el Gobierno Nacional anunció a través de un comunicado de prensa las medidas que se tomaron en ese momento en materia de educación para ofrecer garantías de salud pública a la comunidad. Se dispuso modificar el calendario académico en todo el territorio nacional para preservar el derecho a la salud y a la educación. Para ello se dictaminó lo siguiente:

1. Los docentes y directivos docentes adelantarán actividades de desarrollo institucional con el propósito de organizar y preparar metodologías y escenarios de aprendizaje flexible. Y 
planes de estudio viables para ser implementados desde casa a partir de la semana del 16 de marzo y hasta la semana del 27 de marzo.

2. Propiciar un periodo de vacaciones para los educadores con inicio el 30 de marzo hasta el 19 de abril.

3. Los niños, niñas y jóvenes de escuelas públicas permanecerán en receso durante las fechas en que el cuerpo docente esté de vacaciones y no tendrán clases presenciales.

4. Partiendo de la evolución que se tenga de la epidemiología, desde el 20 de abril el MEN determinará la manera en que se continuará con el calendario académico sin dejar de lado la importancia de protegerse contra el virus.

5. A partir del 16 de marzo la plataforma "Aprender Digital, Contenidos para Todos", estará disponible para que la ciudadanía colombiana pueda acceder a ella. Brindando herramientas para padres de familia y cuidadores que les servirá para trabajar las actividades de los escolares desde casa.

6. Las medidas adoptadas serán socializadas a primera hora del 16 de marzo por medio de una videoconferencia en la que participarán todos los secretarios de educación del país.

7. Los colegios privados deberán ajustar sus calendarios académicos o recurrir a la utilización de las Tecnologías de la Información y la Comunicación (TIC), así como las guías elaboradas para llevar a cabo clases presenciales con base en los lineamientos predispuestos.

La plataforma "Aprender Digital, Contenidos para Todos" ha sido una apuesta para continuar con los procesos educativos al interior de la Nación. En ella, como se describió anteriormente, se brindan herramientas para que los padres y cuidadores de los educandos tengan cómo realizar las actividades asignadas por las instituciones educativas. No obstante, al ser comunicada esta propuesta a través de la Circular No. 19 del 14 de marzo 2020 y de la Circular No. 20 del 16 de marzo de ese mismo año, no se logra apreciar que se hable acerca de la carencia de equipos tecnológicos y de conectividad de los habitantes menos favorecidos del país.

En continuidad, se hizo la búsqueda de información sobre cómo los colegios de carácter privado hicieron uso de las TIC y se halló que, gracias a que durante el segundo semestre del año 2019 se originaron marchas por paro nacional, lo que les afectó, se vieron en la tarea de adelantar e implementar medidas en las que se diera la educación a distancia. Proyecto que hoy tiene un gran éxito no solo a nivel nacional sino a nivel internacional. El Colegio Nueva Granada (CNG) ubicado en la ciudad de Bogotá es uno de esos colegios que tomó la iniciativa de adelantar esta tarea. Y para el 4 de marzo de 2020 ya estaba perfeccionando su protocolo a distancia. Siendo el primer colegio en anunciar el cierre de su Campus a causa de la crisis por COVID-19 mientras que compartió vía open source su protocolo a distancia; quien a la fecha ha obtenido más de once mil visitas.

Al revisar la información básica de esta institución educativa se pudo ver que esta cuenta con los recursos suficientes y quizás más de lo necesario para desarrollar ideas como esta. Puesto que es un colegio privado en la que una matrícula supera el millón de pesos colombiano. Nada que hacer una institución de carácter público ante ella, pues no cuenta con recursos de la misma dimensión. Además, sus estudiantes si acaso poseen las condiciones mínimas para 
subsistir. Esto deja en claro que las diferencias y exclusiones sociales y educativas siguen estando en la mesa como el plato fuerte.

En junio de 2020 se determinaron los lineamientos para la prestación del servicio de educación en casa y en presencialidad bajo el esquema de alternancia y la implementación de prácticas de bioseguridad en la comunidad educativa. Estos buscan adelantar el trabajo que se viene haciendo para dar frente ante las problemáticas educativas presentada a nivel nacional por COVID-19. Pero, hasta la fecha no ha sido posible desarrollarlos todos teniendo en cuenta la igualdad puesto que como se ha venido diciendo, solo aquellas instituciones educativas privilegiadas cuentan con los recursos para brindar clases presenciales en la alternancia mientas que, los menos favorecidos deben seguir buscando estrategias como recurrir a las clases mediante el uso de WhatsApp para no perder el ritmo escolar.

\section{Barreras digitales en la educación colombiana durante la pandemia por COVID-19}

En los últimos meses se ha abierto un abanico correspondiente a la falta de oportunidades para el acceso a la educación, gracias a que, factores incesantes como los sociales, económicos, políticos y culturales han intervenido significativamente en la consumación de una calidad educativa justa, participativa e inclusiva. Así, Noticias ONU, por medio de su publicación el 12 de febrero de 2021, hace ver que los integrantes de una organización sin ánimo de lucro conocida como El Origen están cada vez más convencidos de que la educación digital incluyente es papel fundamental para reducir las brechas económicas, sociales y educativas en todo el mundo. En la misma, se menciona que Tania Rojas, una joven de La Guajira, quien ha considerado la importancia de la inclusión digital, se puso en la tarea de crear una aplicación pensada en las necesidades atribuidas a cada comunidad. De esta manera, ella asevera lo siguiente:

"No basta con darle internet a todo el mundo, hay que crear herramientas específicas según los contextos y que sean personalizables y medibles. Debemos pensar más comunitariamente para crear tecnologías y herramientas globales y no pensar globalmente e intentar impactar a nivel comunitario. Nuestro mensaje es que hay que crear herramientas incluyentes que estén testeadas y basadas en la comunidad para lograr reducir las brechas de forma eficaz".

Lo propuesto por Rojas no está lejos de la realidad, quizá es lo que más se acerca a ella. Como se ha dicho en líneas anteriores, para que exista una verdadera inclusión es urgente partir de las verdaderas necesidades que yacen interna y externamente en cada comunidad puesto que son diferentes la una de la otra. Y si se continúa pensando globalmente e intentando sanar las grietas que no se han analizado desde su raíz misma, el riesgo de acrecentar el círculo que se ha tornado vicioso tomará muchas más fuerza.

Colombia es un país con una diversidad múltiple arraigada en costumbres y creencias que en la mayoría de los casos no dan permiso a ser sobrepasadas. Razón por la que al abordar medidas incluyentes debe promulgarse el hecho de caracterizar y categorizar cada necesidad desde ellas. Cabe decir que las necesidades de quienes tienen NEE, de los afectados por la violencia (cualquiera), de los grupos étnicos, de los habitantes rurales y urbanos son distintas en muchos sentidos. Lo que explica que adoptar las mismas medidas para todos no contribuye a mejorar sino todo lo contrario. Mientras tanto, se sigue hablando de inclusión cuando en realidad lo que se está haciendo es deletrear el vocablo. 
En continuidad, Habemus, en su publicación del 22 de abril de 2020, resalta que "por cuenta de la pandemia de COVID-19, la Presidencia de la República de Colombia planteó una apuesta para no interrumpir la educación en estos tiempos de crisis: la educación virtual. Pero, ¿el país posee la infraestructura tecnológica y las competencias digitales para que la apuesta sea exitosa? De entrada, Colombia tiene una primera barrera para la educación virtual: el acceso a internet"; sin contar que muchas de las personas situadas en zonas urbanas en las que posiblemente la infraestructura tecnológica pudiera ser eficiente, no cuenta con los recursos económicos suficientes para sostener tal servicio. Este, termina diciendo que "aunque la educación virtual supone una esperanza para ampliar la cobertura y mitigar los efectos de la crisis por cuenta del COVID-19, hoy la brecha digital en Colombia es una zancadilla para los planes del Ministerio de Educación y de la Presidencia de la República”.

Zancadilla que le ha costado muy caro a las comunidades educativas con menos recursos económicos y ubicadas en los lugares del plano territorial con menor acceso. Al ser estas la de mayor auge en todo el país los entes gubernamentales debieron crear políticas públicas dirigidas al cierre de estas brechas. Está de más decir que el trabajo a realizar frente a esta situación es bastante demandante y extenso. Y no sirve de nada seguir empleando esfuerzos que quedan en el mero intento.

El Espectador, en la publicación que realizó el pasado 13 de marzo de 2020, indicó que a través del análisis llevado a cabo por el Laboratorio de Economía de la Educación (LEE) de la Universidad Javeriana, este, concluyó "que el 96\% de los municipios en el país no podrían implementar clases virtuales en caso de que se tomara la decisión de cerrar los colegios para evitar contagios, pues más de la mitad de sus estudiantes no tienen acceso a computador ni internet en casa". Es evidente que la cifra abarca casi la totalidad de los municipios del país y, aun cuando se trate de resolver la situación ofreciendo equipos tecnológicos, podría decirse que un bajo porcentaje de estudiantes y familias se beneficiarían de esta iniciativa gracias a que existen hogares en los que hay más de un niño, niña o joven estudiando. Eso sin mencionar que el acceso a internet ya sea por problemas de conectividad o por no contar con los recursos para el pago del servicio ha de ser reducido.

Estefan (2020), hace referencia a dos características con relación a la implementación de una aula virtual como respuesta a la emergencia sanitaria; una de ellas es la conectividad, ya que según él, " el acceso es una de las primeras características que se debe tomar en cuenta para implementar un modelo educativo virtual. Al revisar las cifras de conectividad en la educación básica y superior se encuentran brechas que requieren fuertes inversiones para su reducción. El Departamento Administrativo Nacional de Estadística (DANE) indica que solo alrededor de un $26 \%$ de los estudiantes en zonas rurales tienen conectividad frente a un $89 \%$ en zonas urbanas". Finalmente, hace alusión al modelo de educación virtual, pues, piensa que "la tecnología no debe cumplir una función instrumental para el ejercicio de la práctica docente; la didáctica en la virtualidad implica concebir ritmos de aprendizaje, modificar el rol docente, trabajar tendencias educativas para la mediación tecnológica, flexibilizar la evaluación, entre otros aspectos". He aquí uno de los indicadores más amplios para repensar en la calidad de educación que se está desarrollando. Debido a que, los educadores carecen de capacitaciones constantes que les permitan conocer y enfrentarse directamente a situaciones como esta. En donde el común denominador es la falta de oportunidades desde la base enseñar - docente, aprender - estudiante. 


\section{Conclusiones}

Un sistema escolar de calidad debe cumplir con las expectativas de las necesidades que adolecen las poblaciones a las cuales va dirigido para que hayan garantías en cuanto a la igualdad y la inclusión. Esto a fin de que existan oportunidades para todos y cada uno de los niños, niñas y adolescentes no solo desde el ámbito enseñanza - aprendizaje, adquisición de competencias fundamentales para la vida y el trabajo, y el desarrollo de las habilidades duras y blandas sino también, desde el acceso mismo a la educación que contribuya a la dignidad humana. Lo cual no se refleja en el sistema educativo nacional a pesar de que antes y durante la emergencia sanitaria por COVID-19, el MEN junto a los entes gubernamentales han intentado dar solución a algunas de las problemáticas que permanecen latentes al interior de las comunidades educativas colombianas.

Así, es preciso resaltar que para el Ministerio de Educación Nacional (2016, p. 141) "un sistema escolar básico de calidad" es aquel que ha de brindar garantías para que los niños, niñas y jóvenes logren que su aprendizaje se eleve a su máximo potencial. ¿Qué es lo básico? $\mathrm{Al}$ ser considerado básico, se alcanza a entender que este desde sus inicios la base sobre la que se sostiene es no es lo suficiente sólida. Puesto que solo ha de contar con elementos llanos dejando de lado que las exigencias y demandas de quienes esperan recibir el servicio de educación son superiores conforme pasa el tiempo. De este modo, cabe revisar con detenimiento y a profundidad la percepción que se tiene sobre continuar ofreciendo un sistema de educación básico, para que se dé lugar a nuevas reestructuraciones basadas en el análisis y en la reflexión de las distintas problemáticas que se ilustran.

Las desigualdades sociales, continúan siendo uno de los factores que marcan el estancamiento de los esfuerzos que se emprenden en pro de mejorar el sistema educativo, porque los retos y desafíos que implican la educación son cada vez más disímiles y difíciles de abordar, y mientras se trata de subsanar una área de ella, las demás se ven relegadas haciendo que se deterioren a tal punto de propiciar envergaduras sumamente mayores a la inicial. La pandemia provocada por el Coronavirus dejó ver que un alto porcentaje de estudiantes colombianos padecen de exclusión educativa y social. Gracias a que al momento de tomar las decisiones que dieran paso a continuar con las clases realmente no se hizo un estudio acerca del impacto que generaría el trasladar las aulas a la virtualidad. Es fácil pensar en que al encontrarse la sociedad sumergida en la era tecnológica todas los personas han de poseer un dispositivo que cumpla con los requerimientos que solicitan las clases virtuales.

Se comprobó que factores como el anterior, la conectividad y los recursos económicos que permitieran el pago del servicio internet y la compra de estos equipos desestabilizó grandemente la posibilidad de que los escolares colombianos accedieran a la educación, aun cuando el plan emergente por el MEN se presentó como la mejor opción. Incluso los profesores se vieron afectados, no solo porque el trabajo se les triplicó sino, porque al igual que los escolares no cuentan con equipos tecnológicos, internet o han tenido inconvenientes con la conectividad. Razón por la que han buscado otros métodos para continuar con sus clases. Sin duda, el sistema educativo reclama una reformación que dé el espacio a instaurar con prontitud políticas públicas que logren solventar las heridas causadas por el COVID-19.

La inequidad en el aprendizaje y la probabilidad de que aumenten las deserciones escolares son bastante altas (Moreno, 2020). Cada estudiante es diferente y necesita de un proceso de enseñanza aprendizaje que se ajuste a sus exigencias y necesidades, para que la educación que 
se le preste cumpla con los estándares de calidad. Los educandos con Necesidades Educativas Especiales requieren de herramientas, materiales, acompañamiento y apoyo idóneo que promueva e incentive un aprendizaje significativo y la adquisición de competencias y habilidades que les ayude a desenvolverse con asertividad en los contextos sociales, afectivos, educativos y laborales. Lo que al interior del quehacer pedagógico deja mucho que desear; si bien los maestros no cuentan con el direccionamiento y las herramientas que favorezcan a la creación de estrategias para abordar a esta población desde la presencialidad, hacerlo por medio de la virtualidad acarreará situaciones de incertidumbre y de frustración tanto para ellos, como para los estudiantes y sus padres o cuidadores sin obtener resultados benéficos.

Por último, se destaca que para lograr que la educación en Colombia sea inclusiva en todos sus aspectos, es pertinente partir del fiel compromiso de plantear objetivos claros que labren el camino a seguir para alcanzar la meta de una educación para todos y de alta calidad. Es imperante hacer una revisión exhaustiva en la que se bosquejen soluciones basadas en las realidades que persisten en la actualidad como las consentidas por el Coronavirus, y bajo una mirada futurista de aquellas posibles situaciones que se han de incrementar gracias a los cambios que se dan a nivel socioeconómico, político, cultural y demás, ya que estos viajan a través del tiempo a la velocidad de la luz. El COVID-19 no solo ha aflorado las marcadas desigualdades que permean en la sociedad, también constituye el agravante que ha propiciado un encadenamiento de las falencias que se tienen al relacionar los recursos propuestos con las necesidades educativas, siendo estos poco eficaces y eficientes frente a las problemáticas ya situadas y nacientes. De este modo se advierte que la urgencia de crear y desarrollar políticas que desarraiguen las exclusiones educativas y sociales no debe considerarse netamente a partir de presentar soluciones ante la falta de conectividad y de equipos tecnológicos, es importante abarcar hasta el más mínimo detalle. Pues la población vulnerable no solo es aquella concebida por el MEN, son todos los niños, niñas y jóvenes que reclaman condiciones dignas para acceder y recibir un servicio de educación de alta calidad.

\section{Referencias}

Almenara, J. y Córdoba, M. (2009, marzo). Inclusión educativa: Inclusión digital. Revista Educación Inclusiva, 2,1(61-77) http://rabida.uhu.es/dspace/bitstream/handle/10272/11296/Inclusion_ educativa.pdf?sequence $=2$

Anónimo. (2020, 30 de abril). Así ha afectado el Covid-19 la educación en Colombia. [blog] Forbes Colombia. https://forbes.co/2020/04/30/actualidad/asi-ha-afectado-el-covid-19-la-educacion-encolombia/

Anónimo. (2021, 12 de febrero). Jóvenes colombianos luchan por una educación digital sin barreras sociales ni raciales para los estudiantes indígenas. Noticias ONU https://news.un.org/es/ story/2021/ $02 / 1487982$

Behrman, J. R., A. Gaviria y M. Székely (eds.) (2003). Who’s In and Who’s Out: Social Exclusion in Latin America. Washington, D. C.: Banco Interamericano de Desarrollo.

Bosada, M. (2020, 27 de mayo). La educación emocional, clave para la enseñanza - aprendizaje en tiempos de coronavirus [blog]. Educaweb. https://www.educaweb.com/noticia/2020/05/27/educacionemocional-clave-ensenanza-aprendizaje-tiempos-coronavirus-19205/

Botero, C. (2021, 11 de enero). La pandemia y las brechas digitales [blog]. Razonpublica.com https://razonpublica.com/la-pandemia-las-brechas-digitales/

Cabero, J. (2004). Reflexiones sobre brecha digital. En Soto, F. y Rodríguez, J. (coord.) Tecnología, educación y diversidad: retos y realidades de la inclusión digital. Consejería de Educación y Cultura, (23-42).

Cataldo, M. (2020). Cerrar la brecha digital [blog]. Pacto Global Red Colombia. https://www.pactoglobalcolombia.org/news/cerrar-la-brecha-digital.html 
Comisión Económica para América Latina y el Caribe. 2016. Desarrollo social inclusivo. Una nueva generación de políticas para superar la pobreza y reducir la desigualdad en América Latina y el Caribe. LC.L/4056/Rev.1. CEPAL.

Departamento Nacional de Planeación (2015), Bases Plan Nacional de Desarrollo 2015-2018, Departamento Nacional de Planeación.

Estefan, R. (2020, 20 de mayo). Educación virtual: realidad o ficción en tiempos de pandemia [blog] Pesquisa Javeriana https://www.javeriana.edu.co/pesquisa/educacion-virtual-realidad-o-ficcion-en-tiempos-depandemia/

Fernández A. (2003, septiembre). Educación inclusiva: Enseñar y aprender entre la diversidad. Rev Digit UMBRAL, 13.

Habemus (22/04/2020). La brecha digital en Colombia, ¿una zancadilla para la educación en casa?. Habemus https://habemus.com.co/educacion/ciencias-sociales/cultura/la-brecha-digital-en-colombia/

Hurtado, L. y Agudelo, M. (2014). Inclusión educativa de las personas con discapacidad en Colombia. CES Movimiento y Salud, 2/1(45-55) http://riberdis.cedd.net/bitstream/handle/11181/4889/ Inclusion_ educativa_de_las_personas_con_discapacidad_en_Colombia.pdf?sequence $=1 \& \mathrm{rd}=0031320843818156$

López Melero, M. (1997). Diversidad y cultura: En busca de los paradigmas perdidos. En Arnáiz, P. y De Haro, R. (eds.) 10 años de integración en España: análisis de la realidad y perspectivas de futuro. [Universidad de Murcia], (181-207).

Ministerio de Educación Nacional. (2007, 03 de diciembre). Política pública nacional de primera infancia "Colombia por la primera infancia". MEN. https://www.mineducacion.gov.co/1759/articles177832_archivo_pdf_Conpes_109.pdf

Ministerio de Educación Nacional. (2013). Estrategia de atención integral a la primera infancia FUNDAMENTOS POLÍTICOS, TÉCNICOS Y DE GESTIÓN. MEN. http://www.deceroasiempre. gov.co/QuienesSomos/Documents/Fundamientos-politicos-tecnicos-gestion-de-cero-a-siempre.pdf

Ministerio de Educación Nacional. (2015). OECD-Colombia education and skills accession policy review: Country background report. MEN.

Ministerio de Educación Nacional. (2016). Revisión de políticas nacionales de educación. La educación en Colombia. OCDE http://www.plandecenal.edu.co/cms/media/herramientas/oecd_educacion_en_ colombia.pdf

Ministerio de Educación Nacional (2020a, 14 de marzo) Circular No. 19. MEN. https://www.mineducacion.gov.co/1759/articles-393910_recurso_1.pdf

Ministerio de Educación Nacional (2020b, 15 de marzo). Gobierno Nacional anuncia medidas en materia educativa para ofrecer garantías de salud pública a la comunidad. Mineducación. https://www.mineducacion.gov.co/portal/salaprensa/Noticias/393933:Gobierno-Nacional-anunciamedidas-en-materia-educativa-para-ofrecer-garantias-de-salud-publica-a-la-comunidad

Ministerio de Educación Nacional (2020c, 31 de marzo). Gobierno fortalecerá las competencias digitales en los colegios públicos con la política pública de Tecnologías Para Aprender. Mineducación. https://www.mineducacion.gov.co/1759/w3-article-395101.html?_noredirect=1

Ministerio de Educación Nacional (2020d, junio). lineamientos para la prestación del servicio de educación en casa y en presencialidad bajo el esquema de alternancia y la implementación de prácticas de bioseguridad en la comunidad educativa. MEN. https://www.mineducacion.gov.co/1759/articles399094_recurso_1.pdf

Ministerio de Educación Nacional (2020e, 30 de noviembre). Atención a población vulnerable. Mineducación. https://www.mineducacion.gov.co/portal/Preescolar-basica-y-media/ProyectosCobertura/235112:Atencion-a-poblacion-vulnerable

MinTic (2017). Informe de acciones de política para el cierre de la brecha digital. MinTic https://www.mintic.gov.co/portal/604/articles62254_Documento_de_Cierre.pdf

Mokate, K. (2002), Eficacia, eficiencia, equidad y sostenibilidad: ¿Qué queremos decir? http://www.cepal. org/ilpes/noticias/paginas/9/37779/gover_2006_03_eficacia_eficiencia.pdf

Moreno, V. (2020). El Covid-19 como oportunidad de cambio ante la crisis perpetua de la educación en Colombia. [blog] Universidad Externado de Colombia, Economía https://www.uexternado.edu.co/ economia/senales-boletin-de-coyuntura-y-opinion/el-covid-19-como-oportunidad-de-cambio-ante-lacrisis-perpetua-de-la-educacion-en-colombia/ 
Múñoz-Pogossian, y Barrantes, (s. f.). Equidad e inclusión social: Superando desigualdades hacia sociedades más inclusivas [Preparado por el Departamento de Inclusión Social]. p.; cm. (OAS. Documentos oficiales; OEA/Ser.D/XXVI.15) http://www.oas.org/docs/inclusion_social/equidad-e-inclusionsocial-entrega-web.pdf

OCDE (2015), Education at a Glance Interim Report: Update of Employment and Educational Attainment Indicators, OECD Publishing www.oecd.org/edu/EAG-Interim-report.pdf

Oficina del Alto Comisionado para los Derechos Humanos. (2012). Naciones Unidas, aprobado por la Resolución 21/11 del Consejo de Derechos, p.5. Los Principios Rectores sobre la Extrema Pobreza y los Derechos Humanos.

Padilla, A. (2011, diciembre). Inclusive Education of Persons with Disabilities. Rev Colomb Psiquiatr. 40(4).

Silver, H. (1995). Reconceptualizing Social Disadvantage: Three Paradigms of Social Exclusion. Ginebra: Instituto Internacional de Estudios Laborales, Programa de Desarrollo de las Naciones Unidas.

Redacción Educación. (13 de marzo de 2020). Los colegios públicos de Colombia no están preparados para dar clases virtuales. El Espectador https://www.elespectador.com/coronavirus/los-colegios-publicosde-colombia-no-estan-preparados-para-dar-clases-virtuales-articulo-909149/

Tilly, C. (1999). Durable Inequality. Berkeley, CA: University of California Press.

UNESCO (2005). Informe de seguimiento de la educación para todos. UNESCO.

UNESCO-UIS (2015). Browse by theme: Education. Data Centre, UNESCO Institute for Statistics. www.uis.unesco.org/DataCentre/Pages/BrowseEducation.aspx

UNICEF (2012), Estudio de País: Colombia, Iniciativa Global por las Niñas y los Niños fuera de la Escuela, Fondo de las Naciones Unidas para la Infancia.

White, D. y Le Cornu, A. (2011, 5 de septiembre). Visitantes y Residentes: Una nueva tipología para la participación en línea. First Monday, 16(9-5). 